La Revue

des Droits

de l'Homme

\section{La Revue des droits de l'homme}

Revue du Centre de recherches et d'études sur les droits fondamentaux

Actualités Droits-Libertés | 2016

\title{
Accord entre le Japon et la Corée du Sud concernant les "femmes de réconfort ", une reconnaissance faisant obstacle aux réparations
}

Violences sexuelles (Droit international)

Jérémie Kouzmine

\section{OpenEdition}

Journals

Édition électronique

URL : http://journals.openedition.org/revdh/2081

DOI : $10.4000 /$ revdh.2081

ISSN : 2264-119X

Éditeur

Centre de recherches et d'études sur les droits fondamentaux

Référence électronique

Jérémie Kouzmine, «Accord entre le Japon et la Corée du Sud concernant les « femmes de réconfort », une reconnaissance faisant obstacle aux réparations ", La Revue des droits de l'homme [En ligne], Actualités Droits-Libertés, mis en ligne le 12 mai 2016, consulté le 19 avril 2019. URL : http:// journals.openedition.org/revdh/2081 ; DOl : 10.4000/revdh.2081

Ce document a été généré automatiquement le 19 avril 2019

Tous droits réservés 


\section{Accord entre le Japon et la Corée du Sud concernant les « femmes de réconfort », une reconnaissance faisant obstacle aux réparations}

Violences sexuelles (Droit international)

Jérémie Kouzmine

1 Le terme de «femmes de réconfort » est un euphémisme. C'est le nom que donnaient les militaires de l'armée japonaise à ces femmes enfermées et utilisées comme esclaves sexuelles au cours de la Seconde Guerre Mondiale. ${ }^{1}$ Selon les sources, la pratique a concerné entre 10000 et 200000 femmes. $^{2}$ En Corée du Sud, il ne resterait que 46 survivantes.

2 On estime que de telles pratiques ont émergé en 1932, avec des lieux offrant aux militaires un accès à divers services sexuels, avant que le système ne s'institutionnalise en 1937. ${ }^{3}$ Ces «lieux de réconfort » avaient pour but, selon les autorités, de réduire le nombre de viols dans les zones de stationnement de l'armée, faire chuter la transmission d'infections sexuellement transmissibles, contrecarrer l'espionnage, mais aussi fournir aux soldats une activité récréative, les rapports sexuels permettant supposément d'améliorer leur efficacité au combat. ${ }^{4}$

3 Après plus de 50 ans de silence, les femmes victimes de cet esclavage sexuel ont pris la parole et dénoncé ces faits, ouvrant la voie à un long processus tendant à la réparation des dommages subis. C'est ainsi que, le 28 décembre 2015, le Ministre des affaires étrangères japonais, Fumio Kishida, a reconnu la responsabilité du Japon vis-à-vis de ces cas de violences sexuelles. ${ }^{5}$

4 Cette déclaration, prononcée bien longtemps après les faits, pose la question de l'efficacité des réponses apportées à de tels crimes. Face à des violations systématiques des droits humains, il est nécessaire d'adapter les mécanismes de droit commun responsabilité pénale et réparation du préjudice moral et/ou physique. 
5 La reconnaissance de la responsabilité pénale des auteurs de crimes internationaux est une étape essentielle dans le processus de réparation $\left(1^{\circ}\right)$. Mais le travail de réconciliation sociale et d'apaisement des victimes et de leurs familles peut aussi passer par d'autres voies, à condition d'être mené en collaboration avec les communautés intéressées $\left(2^{\circ}\right)$.

\section{$1 \%$ - Le long parcours de la poursuite pénale des violences sexuelles}

6 Avant d'aborder les difficultés qui entourent la qualification de l'esclavage sexuel, il convient de revenir sur la répression des crimes sexuels en général.

\section{A - Bref historique de la répression des crimes sexuels}

7 Les actes dont ont été victimes les "femmes de réconfort " se sont déroulés dans un contexte bien particulier, celui des conflits armés internationaux. Pour faire face à l'ampleur des crimes commis au cours de la Seconde guerre mondiale, les accords mettant en place le Tribunal de Nüremberg établissent trois types de crimes internationaux : les crimes contre la paix, les crimes de guerre et les crimes contre l'Humanité. Il faut noter qu'aucun de ces deux derniers ${ }^{6}$ ne comprend de références aux crimes sexuels.

Il faudra attendre 1949, avec les Conventions de Genève, puis 1977 avec leurs protocoles, pour que les violences sexuelles dans le cadre des conflits armés soient reconnues. En cas de conflit armé international, le premier protocole additionnel aux Conventions prévoit en effet, de manière générale, la protection de la population civile. ${ }^{7}$ Plus particulièrement, l'article 76 protège les femmes contre les crimes sexuels : «1. Les femmes doivent faire l'objet d'un respect particulier et seront protégées, notamment contre le viol, la contrainte à la prostitution et toute autre forme d'attentat à la pudeur. $»^{8}$

9 Ces pratiques étant prohibées en cas de conflit armé international, il est logique de retrouver cette interdiction dans les textes de droit international pénal, et plus particulièrement dans les statuts du Tribunal pénal international pour le Rwanda (TPIR), du Tribunal pénal international pour l'Ex-Yougoslavie (TPIY) et dans le Statut de Rome.

10 La Cour pénale internationale (CPI), en plus du Statut de Rome qui liste et définit les infractions que la Cour peut connaître, dispose d'un texte apportant des informations précises sur les éléments matériels et intentionnels de chaque crime : "Les Éléments des crimes ". ${ }^{9}$ Ce texte évoque les violences sexuelles en fonction des crimes internationaux auxquels elles se rattachent, c'est-à-dire aux crimes contre 1 crimence ${ }^{10}$ ou aux crimes de guerre $^{11}$, et les définit comme suit :

«L'auteur a commis un acte de nature sexuelle sur une ou plusieurs personnes ou a contraint ladite ou lesdites personnes à accomplir un tel acte par la force ou en usant à l'encontre de ladite ou desdites ou de tierces personnes de la menace de la force ou de la coercition, telle que celle causée par la menace de violences, contrainte, détention, pressions psychologiques, abus de pouvoir, ou bien à la faveur d'un environnement coercitif, ou encore en profitant de l'incapacité desdites personnes de donner leur libre consentement $»^{12}$.

11 Le TPIR et TPIY ne disposent pas d'un tel texte et ont dû développer, dans leur jurisprudence, leurs propres définitions. Ainsi, on observe des approches différentes entre les deux tribunaux ad hoc. Alors que le TPIR a abordé les crimes sexuels sous l'angle des crimes contre l'humanité et crimes de génocide, le TPIY les a classés dans la catégorie 
des crimes de guerre. Cette particularité est toutefois certainement liée au type de contentieux qu'ont eu à traiter les deux tribunaux, le TPIR ayant un contentieux lié au génocide et celui du TPIY étant lié aux crimes commis à l'occasion des conflits en ExYougoslavie.

12 Surtout, les violences sexuelles commises dans le cadre de conflits armés poursuivent généralement deux objectifs qui font échos à cette dualité d'incrimination : les violences sexuelles sont, d'une part, une tactique pour anéantir délibérément des vies et terroriser le camp opposé ; mais elles peuvent être utilisées, d'autre part, comme un "carburant " pour les soldats, moteurs de la machinerie de guerre. ${ }^{13}$

13 Le TPIR a d'abord voulu aborder les violences sexuelles sous la qualification de crimes de guerre. Malgré la volonté des procureurs du TPIR de poursuivre les violences sexuelles en tant qu'atteinte à la dignité de la personne, la qualification de violences sexuelles a toujours été écartée au motif que celles-ci n'avaient pas de lien avec le conflit armé, comme il l'est précisé dans l'affaire Akayesu de $1998 .{ }^{14}$ Concernant le viol, il est surprenant qu'aucune condamnation n'ait été prononcée alors que l'article 3 du Statut du TPIR considère expressément ce type d'acte comme constitutif d'un crime de guerre. En revanche, le juge du TPIR qualifie les violences sexuelles sous la forme du viol comme constitutives de crime contre l'humanité au regard de l'article 3 du Statut, ou bien en tant qu'acte constitutif de génocide.

Quant au TPIY, il s'est confronté à la définition du viol dans l'affaire Furundzija en 1998. Le juge y définit d'abord la torture, avant d'y inclure le viol dans le cadre des détentions et interrogatoires : «Le viol est alors utilisé par la personne chargée d'interroger le ou la détenue ou par les autres personnes associées à l'interrogatoire comme un moyen de punir, d'intimider, de contraindre ou d'humilier la victime ou encore d'obtenir d'elle ou d'une tierce personne des renseignements ou des aveux. ${ }^{15}$ Ce n'est qu'ensuite que le TPIY a envisagé le viol de façon autonome en expliquant que, selon les circonstances, le viol peut constituer un crime distinct de la torture. ${ }^{16}$

15 Il faut noter le travail important de mouvements féministes qui, au moment des négociations pour adopter la résolution mettant en place le TPIY, ont lutté pour que le viol soit inscrit explicitement dans le texte et qu'il soit effectivement un chef d'accusation utilisé pour les poursuites ; leur objectif final étant l'inscription du viol en tant que crime international autonome, non soumis à une catégorie de crime plus large. ${ }^{17}$

Concernant plus précisément les crimes commis sur le continent asiatique au cours de la Seconde Guerre Mondiale, il est nécessaire d'analyser la charte ayant créé le Tribunal militaire international pour l'Extrême-Orient. Celle-ci, promulguée le 19 janvier 1946, prévoit trois types de crimes : les crimes contre la paix, les crimes de guerre et les crimes contre l'humanité. Ces derniers comprennent notamment le meurtre, l'extermination, l'esclavage, la déportation et tout " autre acte inhumain commis contre la population civile ». Toutefois, aucune condamnation pour violences sexuelles ou esclavage sexuel n'a été prononcée.

\section{B - La difficulté à poursuivre les faits d'esclavage sexuel}

17 En 1995, le Rapporteur spécial chargé de la question de la violence contre les femmes a publié un rapport suite à une visite en Corée du Nord, Corée du Sud et au Japon. À cette occasion, le rapporteur a déclaré que "l'usage des "femmes de réconfort» devrait être 
considéré comme un cas d'esclavage sexuel et de pratiques équivalentes à l'esclavage, conformément à l'approche adoptée pour dans les institutions et traités internationaux des droits humains $» .^{18}$ personne actuellement poursuivie est accusée d'esclavage sexuel. L'audience de confirmation des charges a permis de préciser quelques éléments constitutifs du crime. Par exemple, l'emprisonnement, tant dans son principe que dans sa durée, ne suffit pas pour constituer l'élément d'exercice des pouvoirs associés au droit de propriété, malgré l'existence concomitante de crimes sexuels. ${ }^{20}$ En revanche, le fait de réduire une personne à la condition d'animal - notamment en appelant les individus de la sorte, et en les forçant à transporter des biens pillés - tout en obligeant ces personnes à subir des actes sexuels, est constitutif du crime d'esclavage sexuel. ${ }^{21}$

Mais force est de constater que la reconnaissance des infractions sur le plan juridique ne suffit pas, et qu'un véritable travail de réparation, en collaboration avec les victimes, est nécessaire.

\section{$2 \%$ Le combat inachevé pour la réparation des crimes subis par les « femmes de réconfort »}

Le processus de réparation des crimes subis par les "femmes de réconfort» a non seulement été soumis aux aléas des relations diplomatiques entre le Japon et la Cirée du sud, mais a aussi conduit à la contestable mise à l'écart de la société civile. 


\section{A - Une réparation soumise aux aléas diplomatiques}

25 À la fin de la guerre, un long processus de réparation a été entamé avec la signature des Accords de Paix à San Francisco en 1951. Conformément à l'article 14 de ces accords, le Japon devait dédommager les Alliés pour les «souffrances et dommages causés pendant la guerre ». Cette obligation était cependant très vite limitée par le même article qui prenait en compte la situation économique du pays en obligeant, dans un premier temps, le Japon à entrer en négociation avec les Etats colonisés au cours de la guerre - ce qui inclut les pays où la pratique des « femmes de réconfort » a existé.

26 Le processus de conciliation a démarré en 1965, dans un souci d'apporter une réponse rapide à ce sujet afin que les futures générations n'héritent pas de cet épineux problème. 10 ans après la fin de la Guerre, le Gouvernement japonais proposait alors de créer un fond de 800 millions de yens.

Entretemps, le droit international a évolué et intégré de façon significative le principe de réparation pour les victimes, comme l'adoption des Principes fondamentaux et directives concernant le droit à un recours et à réparation des victimes de violations flagrantes du droit international des droits de l'Homme et de violations graves du droit international humanitaire (Principes sur les réparations des Nations unies), qui « ont permis de définir une réparation intégrale, qui répond seule effectivement aux droits et besoins des victimes, définition désormais acceptée comme faisant droit en droit international $\gg{ }^{22}$

Il faudra pourtant attendre 1993 pour que les premières excuses officielles soient prononcées, et elles étaient loin d'être satisfaisantes. En effet, le Secrétaire général adjoint au gouvernement de l'époque, Yohei Kono, avait certes reconnu les faits mais en attribuait la cause aux cocontractants privés de l'armée, et non pas à l'armée elle-même.

C'est finalement le 28 décembre 2015 que la Corée du Sud a accepté l'accord du Ministre des affaires étrangères japonais, Fumio Kishida, sous la forme d'un billion de yens pour un fonds dédié aux victimes et des excuses officielles de la part du Premier ministre, Shinzo Abe. À cette occasion, le Ministre des affaires coréen, Yun Byung-se, a déclaré que le conflit diplomatique était résolu de manière définitive et irréversible. ${ }^{23}$

Cependant, aujourd'hui, des articles publiés dans la presse japonaise remettent en question l'existence de la pratique de l'esclavage sexuel par l'armée japonaise et, partant, l'existence même des «femmes de réconfort». Selon les propos tenus par Shinsuke Sugiyama, adjoint au Ministre des affaires étrangères, le 16 février 2016 devant le Comité sur l'élimination de la discrimination à l'égard des femmes, le Japon «n'aurait pas trouvé de documents confirmant que des Comfort women ont été recrutées de force par les militaires ou le gouvernement $\gg .^{24}$

\section{B - Un accord politique contesté par la société civile}

Après plusieurs décennies de silence, les «femmes de réconfort » ont pris la parole. En commençant par Kim Hak-soon, qui, pour la première en août 1991, a abordé publiquement le sujet..$^{25}$ Dès lors, de nombreuses voix se sont élevées pour raconter le vécu de ces femmes. Elles se sont regroupées, ont créé des associations, et ont organisé des manifestations - la plus visible d'entre elles étant un rassemblement chaque mercredi depuis le 8 janvier 1992 devant l'ambassade japonaise à Séoul. ${ }^{26}$ Elles ont également exercé 
plusieurs recours, notamment directement devant les juridictions japonaises, mais aussi devant les tribunaux américains. Chaque recours s'est soldé par un échec. ${ }^{27}$

Une autre initiative importante a été la tenue, du 8 au 12 décembre 2000, du «Tribunal international sur les crimes de guerre contre les femmes et l'esclavage sexuel par l'armée japonaise ", sur l'initiative de l'association VANET (Violence against Women in War Network). Ce tribunal a été mis en place pour plusieurs raisons : répondre au vœu de nombreuses survivantes pour que soient jugés les responsables du système des «centres de réconfort », mais aussi rendre justice aux victimes laissées de côté, mener une analyse sur les causes profondes qui avaient permis l'impunité du crime, et enfin dévoiler les dommages subis par des « femmes de réconfort » japonaises. ${ }^{28}$

Dans ce contexte, l'accord du 28 décembre 2015 a essuyé un certain nombre de critiques de la part de la société civile.

"Cet accord s'avère problématique, à commencer parce que les survivantes n'ont pas été consultées. Par ailleurs, si le premier ministre japonais, longtemps révisionniste sur cette question, a présenté ses «excuses et son repentir, du plus profond de son cœur", le gouvernement se garde bien de reconnaître sa responsabilité juridique. Enfin, l'éducation et la revendication d'éduquer les générations futures pour éviter la répétition de tels faits a été totalement occultée, ce qui nuit incontestablement au travail de mémoire ${ }^{29}$

Il est ainsi possible de dégager trois éléments :

La première critique concerne le fait que la société civile n'ait justement pas été consultée. Dans des négociations concernant la réparation, il est indispensable d'inclure ces mêmes victimes dans les discussions. Cela n'a pas été le cas pour la négociation entre le Japon et la Corée du Sud, malgré l'existence d'associations de victimes. ${ }^{30}$

La deuxième limite concerne la nature du texte. Il ne s'agit que d'un accord diplomatique, qui ne pose pas la question de la responsabilité juridique des bourreaux, parfois encore vivants. L'impunité est pourtant le premier obstacle à la justice et au processus de réparation. Cette critique est d'autant plus forte que les accords ont été présentés comme étant définitifs et irréversibles, ce qui ferme la porte à la mise en jeu d'une telle responsabilité.

37 Enfin, le dernier problème que pose cet accord concerne une demande particulière, et que l'on retrouve pour d'autres conflits d'une telle ampleur : l'éducation. Les livres d'histoire actuels occultent entièrement l'existence des «femmes de réconfort ». Il avait été donc demandé de revoir les programmes scolaires. Il apparaît essentiel d'enseigner cette partie de l'Histoire pour les futures générations.

Les Lettres « Actualités Droits-Libertés » (ADL) du CREDOF (pour s'y abonner et se désabonner) sont accessibles sur le site de la Revue des Droits de l'Homme (RevDH) Contact 


\section{NOTES}

1. Description des missions de l'association «Washington Coalition for Comfort Women Issues ».

2. BBC, " "Comfort women" - a painful legacy for Tokyo and Seoul », 28 décembre 2015.

3. Amnesty international, Japan - STILL WAITING AFTER 60 YEARS : JUSTICE FOR SURVIVORS

OF JAPAN'S MILITARY SEXUAL SLAVERY SYSTEM, 2005, p. 6.

4. Ibidem.

5. http://jurist.org/paperchase/2015/12/south-korea-and-japan-resolve-comfortwomen-issue.php.

6. Art. 6(b) Accord concernant la poursuite et le châtiment des grands criminels de guerre des Puissances européennes de l'Axe et statut du tribunal international militaire, Londres, 8 août 1945: ' Les Crimes de Guerre ': c'est-à-dire les violations des lois et coutumes de la guerre. Ces violations comprennent, sans y être limitées, l'assassinat, les mauvais traitements et la déportation pour des travaux forcés ou pour tout autre but, des populations civiles dans les territoires occupés, l'assassinat ou les mauvais traitements des prisonniers de guerre ou des personnes en mer, l'exécution des otages, le pillage des biens publics ou privés, la destruction sans motif des villes et des villages ou la dévastation que ne justifient pas les exigences militaires.

7. Art. 51 du Protocole additionnel aux Conventions de Genève du 12 août 1949 relatif à la protection des victimes des conflits armés internationaux (Protocole I), 8 juin 1977.

8. Art. 76 al. 1 du Protocole additionnel aux Conventions de Genève du 12 août 1949 relatif à la protection des victimes des conflits armés internationaux (Protocole I), 8 juin 1977.

9. Zappala, S., "Article 9 - Éléments de crimes ", Statut de Rome de la Cour pénale internationale, commentaire article par article, Sous la direction de J. Fernandez, X. Pacreau et la coordination éditoriale de L. Maze Editions Pedone, Paris, 2012.

10. Jurovics, Y.., "Article 7 - Crimes contre l'humanité", Statut de Rome de la Cour pénale internationale, commentaire article par article, Sous la direction de J. Fernandez, X. Pacreau et la coordination éditoriale de L. Maze Editions Pedone, Paris, 2012.

11. Eudes, M., "Article 8 - Crimes de guerre", Statut de Rome de la Cour pénale internationale, commentaire article par article, Sous la direction de J. Fernandez, X. Pacreau et la coordination éditoriale de L. Maze Editions Pedone, Paris, 2012.

12. Articles 7 1) g) $-6,8$ 2) b) xxi-6, 8 2) e) vi)-6, Eléments des crimes, Documents officiels de l'Assemblée des États Parties au Statut de Rome de la Cour pénale internationale, première session, New York, 3-10 septembre 2002.

13. ASKIN, K.D., "The quest for post-conflict gender justice ", Columbia Journal of Transnational Law, 2002-2003, p. 511.

14. TPIR, Le Procureur c. Jean-Paul Akaysu, Chambre I, No. ICTR-96-4-T, 2 septembre 1998, $\$ 643$.

15. TPIY, Le Procureur c. Anto Furundzija, Chambre de première instance, No. IT-95-17/1-T, 10 décembre 1998, §163. 
16. Ibidem, $\$ 164$.

17. HALLEY, J., « Rape in Berlin: Reconsidering the criminilisation of rape in the international law of armed conflict », Melbourne Journal of International Law, vol. 9, 2008, p. 5.

18. Conseil économique et social des Nations Unies, Rapporteur spécial chargé de la question de la violence contre les femmes, Report on the mission to the Democratic People's Republic of Korea, the Republic of Korea and Japan on the issue of military sexual slavery in wartime, E/CN.4/1996/53/Add.1, 4 janvier 1996, §8.

19. La Cour n'a une compétence que pour les actes commis postérieurement à l'entrée en vigueur du Statut de Rome, soit le 1er juillet 2002.

20. CPI, Le Procureur c. Bosco Ntaganda, Chambre préliminaire II, No. ICC-01/04-02/06, 9 juin 2014, §53.

21. Ibidem, $\S \S 54,55,56$ et 57 .

22. FIDH, RDC : Les victimes de crimes sexuels obtiennent rarement justice et jamais réparation, Octobre 2013, p. 34.

23. http://jurist.org/paperchase/2015/12/south-korea-and-japan-resolve-comfortwomen-issue.php

24. http://www.japantimes.co.jp/news/2016/02/17/national/history/no-documentsfound-confirm-military-forced-comfort-women-sexual-servitude-japan-envoy-tells-u-n/ \#.VuQhSs5FRM0

25. Amnesty international, Japan - STILL WAITING AFTER 60 YEARS: JUSTICE FOR SURVIVORS OF JAPAN'S MILITARY SEXUAL SLAVERY SYSTEM, 2005, p. 17.

26. Amnesty international, Japan - STILL WAITING AFTER 60 YEARS : JUSTICE FOR SURVIVORS OF JAPAN'S MILITARY SEXUAL SLAVERY SYSTEM, 2005, p. 6.

27. Ibidem.

28. NISHINO, R., « Le tribunal d'opinion de Tôkyô pour les « femmes de réconfort » », Droit et cultures [En ligne], 58 | 2009-2.

29. Claire San Filippo, Responsable Droit des femmes et Droits des Migrants de la Fédération internationale des ligues de droits de l'Homme.

30. Deutsche Welle, «'Comfort women' - The wounds of their lives », 15 janvier 2016.

\section{RÉSUMÉS}

Le 28 décembre 2015, le Ministre des affaires étrangères japonais, Fumio Kishida, a reconnu au nom de son gouvernement la responsabilité du Japon dans les faits de violences sexuelles commis à partir de 1932 et particulièrement au cours de la Seconde Guerre mondiale, par les soldats de l'armée japonaise envers des femmes coréennes, chinoises et philippines. Cette déclaration, à la fois diplomatique et symbolique, s'accompagne de la création d'un fonds pour les victimes, doté d'un billion de yens. Si la lutte de ces "femmes de réconfort » pour obtenir réparation dure depuis plusieurs décennies, la réponse apportée par le gouvernement japonais est pourtant loin d'être pleinement satisfaisante. 
AUTEUR

JÉRÉMIE KOUZMINE

Elève-avocat à l'HEDAC 